\title{
What is the optimal treatment strategy for chronic obstructive pulmonary disease exacerbations?
}

\author{
W. Willaert, M. Daenen, P. Bomans, G. Verleden, M. Decramer
}

What is the optimal treatment strategy for chronic obstructive pulmonary disease exacerbations? W. Willaert, M. Daenen, P. Bomans, G. Verleden, M. Decramer. (C) ERS Journals Ltd 2002.

ABSTRACT: The present study aims to determine whether treating chronic obstructive pulmonary disease (COPD) exacerbations with intravenous steroids and aerosol bronchodilators (group I) is superior to oral steroids and multiple dose inhaler (MDI) bronchodilators with a spacer (group II).

Group I received $40 \mathrm{mg}$ methylprednisolone $\cdot \mathrm{day}^{-1}$ intravenously with a decrease to $20 \mathrm{mg}$ after 10 days and a further decrease of $4 \mathrm{mg} \cdot 4 \mathrm{days}^{-1}$. Aerosol therapy consisted of $10 \mathrm{mg}$ salbutamol and $1 \mathrm{mg}$ ipratropiumbromide day $^{-1}$. Group II received $32 \mathrm{mg}$ methylprednisolone orally for 1 week followed by $24 \mathrm{mg} \cdot \mathrm{day}^{-1}$ for 4 days and a

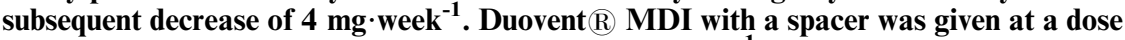
of $1.6 \mathrm{mg}$ fenoterol and $640 \mu \mathrm{g}$ ipratropiumbromide $\cdot \mathrm{day}^{-1}$.

In group I ( $n=19)$ forced expiratory volume in one second (FEV1) rose from $0.82 \pm 0.46$ to $0.91 \pm 0.47 \mathrm{~L}$ and average dyspnoea decreased from $6.0 \pm 1.9$ to $4.1 \pm 2.6$ within 10 days. The Chronic Respiratory Disease Index Questionnaire (CRQ) score increased from $78 \pm 24$ to $90 \pm 24$ points after 4 weeks. In group II (n=18) FEV1 increased from $0.70 \pm 0.27$ to $0.90 \pm 0.29 \mathrm{~L}$, dyspnoea regressed from $6.2 \pm 2.4$ to $2.7 \pm 2.6$ and $\mathrm{CRQ}$ from $67 \pm 17$ to $86 \pm 20$. Both groups showed similar results in dropout rate, length of hospital stay and patient satisfaction.

In conclusion, the two treatment strategies appear equally effective in treating chronic obstructive pulmonary disease exacerbations, although oral steroids and metered dose inhaler bronchodilators appear associated with a higher risk of hospital re-admission.

Eur Respir J 2002; 19: 928-935.
Respiratory Division, University Hospital Gasthuisberg, Katholieke Universiteit Leuven, Belgium.

Correspondence: W. Willaert, Laboratory of Pneumology, Respiratory Muscle Research Unit, Onderwijs \& Navorsing, University Hospital Gasthuisberg, Herestraat 49, B-3000 Leuven, Belgium.

Fax: 3216347126

E-mail: wimwillaert@hotmail.com

Keywords: Aerosols, chronic obstructive pulmonary disease, corticosteroids, exacerbation, metered dose inhaler bronchodilators, treatment

Received: August 32001

Accepted after revision December 7 2001

This study was supported by the Research Foundation Katholieke Universiteit Leuven grant \#OT98/27 and the "Fonds voor Wetenschappelijk Onderzoek Vlaanderen" grants \#G.0/ 75.99 and \#G.0237.01.
Systemic corticosteroids have become a standard component in the pharmacotherapy of chronic obstructive pulmonary disease (COPD) exacerbations and studies have concentrated on their therapeutic effect, optimal dosage, route of administration, time-course of response and adverse effects. These trials and studies have shown the benefit of systemic corticosteroids, administered orally or intravenously, in the emergency treatment of COPD exacerbations. Systemic corticosteroids improve forced expiratory volume in one second (FEV1) [1-6], peak expiratory flow $[4,6]$, oxygen tension in arterial blood [5], subjective dyspnoea symptoms [2, 7], quality of life [2], treatment failure rate [3] and prevention of relapses [8]. Notwithstanding the increase in the number of trials in the last decade evaluating the effect of steroids in COPD, little research has been done to compare orally to intravenously administered steroids when treating an exacerbation in casualty or in patients admitted to hospital.

More attention has been given to comparing wet nebulizers to metered dose inhaler (MDI) as a device for bronchodilator delivery in COPD. A meta-analysis by TURNER et al. [9] and numerous other trials have shown the two devices to be equally effective in the treatment of stable [1-12] and acute [9] COPD in inand outpatient settings. No difference was reported in the effect of bronchodilation, in pulmonary function tests (PFTs), exercise performance, symptom scores or extra $\beta_{2}$-agonist use. The degree of bronchodilation was considered to be a reflection of the administered dose rather than the mode of administration.

There is no consensus on the optimal route of administration for steroids and the device for bronchodilators. Therefore, a prospective, randomized, controlled trial was conducted to investigate the hypothesis that treatment of COPD exacerbations with oral corticosteroids and MDI bronchodilators, compared to intravenous corticosteroids and aerosol bronchodilators, would not result in different outcome variables. Evidence to support this hypothesis would enhance the potential for home treatment of COPD exacerbations. Primary end-points were recovery of FEV1 and forced vital capacity (FVC), subjective dyspnoea, improvement in quality of life, length of hospital stay, need for additional steroids and bronchodilation, patient satisfaction, probability of therapy failure, and hospital re-admission. 


\section{Patients and methods}

\section{Patients}

The inclusion criteria for the study were established before the trial and strictly applied. In the period from July 1999-March 200048 patients with a clinical history of COPD [13] who presented with an acute exacerbation to the casualty department of the University Hospital Gasthuisberg, Leuven, Belgium, were eligible for inclusion in the trial. As in most published definitions, an acute exacerbation was defined as: 1) increased dyspnoea, 2) increased cough frequency or severity, 3) increased production or purulence of sputum, 4) increased wheeze, lasting for at least 3 days and for which the patient sought medical attention. The American Thoracic Society (ATS) indications for hospitalization of patients with COPD exacerbations were applied [13].

Patients were excluded when: 1) there was a personal or family history of asthma (defined as episodic wheezing or dyspnoea that rapidly improved with treatment) or atopy, 2) invasive or noninvasive assisted ventilation was deemed necessary according to the attending casualty physician, 3) the patient was unable to successfully use a MDI as a device for administering bronchodilators.

Patients who had used inhaled or systemic corticosteroids prior to admission to casualty were not excluded. The cumulative dose over a period of 2 weeks prior to admission was recorded and used as a variable in the evaluation of the patients characteristics. The patient characteristics at inclusion are summarized in table 1.

The decision to discharge from hospital was based on physician judgement (after a minimum of a 10-day inpatient therapeutic regime). The Hospital Ethics Committee approved the study protocol.

\section{Study design}

Admission. On admission to casualty, patients were randomly assigned to one of the two therapy groups: Group I received $40 \mathrm{mg}$ methylprednisolone $\cdot$ day $^{-1}$ intravenously with a decrease to $20 \mathrm{mg}$ after 10

Table 1.-Baseline characteristics of the study population

\begin{tabular}{|c|c|c|c|}
\hline Value & Group I & Group II & p-value \\
\hline Subjects $n$ & 23 & 25 & \\
\hline $\mathrm{M}: \mathrm{F} \mathrm{n}$ & $21: 2$ & $21: 4$ & \\
\hline Age yrs & $72 \pm 6$ & $71 \pm 8$ & $>0.5$ \\
\hline Smoking pack-yrs & $27 \pm 16$ & $36 \pm 18$ & $>0.15$ \\
\hline BMI $\mathrm{kg} \cdot \mathrm{m}^{-2}$ & $23.2 \pm 4.4$ & $24.2 \pm 5.4$ & $>0.5$ \\
\hline Baseline FEV1 L & $1.14 \pm 0.43$ & $1.10 \pm 0.51$ & $>0.8$ \\
\hline Baseline FVC L & $2.48 \pm 0.64$ & $2.55 \pm 0.63$ & $>0.7$ \\
\hline Baseline $\mathrm{FEV} 1 / \mathrm{FVC}$ ratio & $47 \pm 16$ & $42 \pm 12$ & $>0.2$ \\
\hline $\begin{array}{l}14 \text { day pre-admission } \\
\text { steroid use mg }\end{array}$ & $98 \pm 89$ & $111 \pm 119$ & $>0.7$ \\
\hline
\end{tabular}

Data are presented as mean \pm SD unless otherwise stated. BMI: body mass index; FEV1: forced expiratory volume in one second; FVC: forced vital capacity; M: male; F: female. days and subsequent oral treatment with a further decrease of $4 \mathrm{mg} \cdot 4$ days $^{-1}$. Aerosol therapy consisted of $10 \mathrm{mg}$ salbutamol.day ${ }^{-1}$ and $1 \mathrm{mg}$ ipratropiumbromide $\cdot \mathrm{day}^{-1}$, administered in 4 aerosols; Group II received $32 \mathrm{mg}$ oral methylprednisolone for 1 week followed by $24 \mathrm{mg} \cdot \mathrm{day}^{-1}$ for a period of 4 days and a subsequent decrease of $4 \mathrm{mg} \cdot \mathrm{week}^{-1}$. Duovent $\left(\mathbb{R}\right.$ was given in $4 \times 4$ puffs $\cdot$ day $^{-1}$ with a cumulative dose of $1.6 \mathrm{mg}$ fenoterol $\cdot \mathrm{day}^{-1}$ and $640 \mu \mathrm{g}$ ipratropiumbromide $\cdot \mathrm{day}^{-1}$, generated from a MDI and delivered with a spacer.

On admission, the patient's health related quality of life was assessed using the Chronic Respiratory Disease Index Questionnaire (CRQ) [14]. Scores for dyspnoea, fatigue, emotional functioning and mastery were determined with the emphasis on the 2 weeks preceding casualty admission. The cumulative 14-day dose of received systemic steroids prior to admission was also noted.

Daily measurements. Daily measurements were performed for a period of 10 days. Bedside spirometry was measured in an upright position at a fixed time $(11: 00 \mathrm{~h})$ using a computerized, portable spirometer (Micro spirometer $\AA$; Micro Medical Limited, Gillingham, UK). The best FEV1 and FVC after three reproducible measurements were used $[15,16]$ in the analysis.

Every day the level of dyspnoea was recorded on a $10 \mathrm{~cm}$ visual analogue scale (VAS). The response could vary from $0 \mathrm{~cm}$ (no shortness of breath) to $10 \mathrm{~cm}$ (severe shortness of breath). Daily use and additional need of bronchodilators and steroids were also recorded.

The respiratory physicians in charge of the patients, who were not part of the investigative team, were allowed to change bronchodilator and steroid dose or administer antibiotics during the trial. They were also free to withdraw patients from the study if they felt clinical improvements were not satisfactory. The physician decided at which point the patient was fit to be discharged (after at least 10 days of therapy). The physicians in charge received no information on the objectives or specific target variables of the trial, thereby minimising bias, although they could not be completely blinded.

Treatment failure was considered when: 1) a patient required invasive or noninvasive assisted ventilation, 2) a patient's clinical improvement was not satisfactory and a change of treatment was deemed necessary by the treating physician, 3) a patient was not satisfied with his or her own clinical progress and demanded another treatment regime (noncompliance).

Discharge. On discharge the length of hospital stay and cumulative steroid dose were recorded, as well as patient satisfaction with the received treatment. To assess the latter the patient could use a score ranging from 1 (not satisfied) to 5 (very satisfied).

Follow-up. Four weeks after discharge the patients returned to the hospital where spirometry and the CRQ were repeated. The satisfaction with the received treatment was also re-evaluated. Probability 
of re-admission to the hospital was evaluated during a 20-week period.

\section{Statistical analysis}

Baseline characteristics of the two therapy groups were compared using unpaired t-tests. T-tests (paired and unpaired) were used to test significance of treatment effects within the groups and between them. Interactions between treatment and time were analysed with repeated measures analysis of variance (ANOVA). Re-admission curves were compared using the log-rank test. Significance level was set at $\mathrm{p}<0.05$. Data are shown as means \pm sD unless otherwise indicated.

\section{Power analysis}

In accordance with previous publications [2, 3] an expected difference between the two treatment strategies of $200 \pm 150 \mathrm{~mL} \mathrm{FEV1}$, was used to compute the sample size. Using a power of $80 \%$ with an $\alpha=0.05$ (two tailed) each treatment strategy would have to consist of nine patients.

A minimal clinically important difference of $10 \pm 10$ points $[17,18]$ in the CRQ score meant that after power analysis 16 patients were required in each treatment group.

To choose an appropriate sample size regarding probability of hospital re-admission, power analysis comparing two proportions was used. An average relapse of 50\% [19] after 6 months with a difference of $20 \%$ [8] required 99 patients in each treatment group to obtain a power of $80 \%$ with an $\alpha=0.05$ (two tailed).

The present study was thus powered to demonstrate differences in FEV1, quality of life, but not in the re-admission rate.

\section{Results}

\section{Study population: intake and follow-up}

Forty-eight eligible patients fulfilled inclusion criteria and were randomized in the trial between July 1999-March 2000 (group I: intravenous steroids and aerosols, $n=23$, group II: oral steroids and MDI bronchodilators, $\mathrm{n}=25$ ).

In group I, four patients dropped out of the trial: three patients had to be admitted to the intensive care unit (ICU) due to clinical deterioration, heart failure, respiratory insufficiency and hypercapnia and one patient suffered a femur fracture and had to be withdrawn. In group II, seven patients dropped out: one patient had to be admitted to the ICU, in two patients progress was deemed unsatisfactory by the attending physician, three patients were not satisfied with the received treatment (noncompliance) and one patient was diagnosed with a lung tumour.

No statistically significant difference in dropout rate between the two groups could be seen. In group I, seven patients were lost in the 4-week follow-up: two were readmitted prior to follow-up, one patient died and four patients refused to attend. In group II, nine patients were lost: two were re-admitted, six patients refused to attend and one patient could not successfully undergo the CRQ as he was not able to understand the asked questions.

As can be seen in table 1, patients had severe COPD. At intake there were no significant differences between the two groups. Age, smoking history, body mass index and baseline pulmonary function tests were similar in both groups. More importantly, there was no difference in 14-day cumulative systemic steroid use prior to admission to casualty: group I had received $98 \pm 89 \mathrm{mg}$ and group II $111 \pm 119 \mathrm{mg}$ $(\mathrm{p}=0.74)$.

\section{Bedside spirometry}

Ninety per cent of all planned spirograms were obtained. Two of the obtained spirograms $(0.6 \%)$ were of insufficient quality and could not be used in the analysis.

In group I the FEV1 rose from $0.82 \pm 0.46 \mathrm{~L}$ $(32 \pm 16 \%$ predicted) on day $1-2$ to $0.91 \pm 0.47 \mathrm{~L}$ (35 $\pm 17 \%$ pred) on day 9-10 (an increase of $22 \pm 61 \%$, $\mathrm{p}=0.19)$. Using the repeated measures ANOVA test, no significant interaction between treatment and time was demonstrated $(\mathrm{p}=0.99)$. The FEV1 of patients who attended the 4-week follow-up visit increased from $0.89 \pm 0.34 \mathrm{~L}(36 \pm 13 \%$ pred $)$ on day 10 to $0.91 \pm 0.35 \mathrm{~L}$ $(37 \pm 14 \%$ pred) at 4 -weeks (an increase of $5 \pm 24 \%$, $\mathrm{p}=0.79)$.

Group II showed a 10-day increase from $0.70 \pm 0.27$ $(29 \pm 11 \%$ pred) to $0.90 \pm 0.29 \mathrm{~L}(35 \pm 10 \%$ pred) (an increase of $35 \pm 32 \%, \mathrm{p}=0.0005)$. Repeated measures ANOVA, however, revealed no significant interaction in time $(\mathrm{p}=0.62)$. After 4 -weeks of follow-up, FEV1 had increased from $1.12 \pm 0.35(42 \pm 10 \%$ pred $)$ to $1.31 \pm 0.40 \mathrm{~L}(50 \pm 13 \%$ pred) (an increase of $20 \pm 33 \%$, $\mathrm{p}=0.091$ ). None of the changes in FEV1 differed significantly between the two groups: the per cent increase after 10 days $(p=0.5)$, daily increase and per cent follow-up change $(p=0.29)$ followed a similar evolution.

The changes in FVC resembled FEV1, as can be seen on figure 1 . No statistically significant difference could be shown between the two groups. The means and mean per cent changes in FEV1 and FVC for group I and II during the 10 days of measurements are given in figure 1 and table 2.

\section{Dyspnoea}

At the 10-day measurement point, dyspnoea had decreased from $6.0 \pm 1.9$ to $4.1 \pm 2.6$ on the VAS (a decrease of $30 \pm 42 \%, \mathrm{p}=0.004$ ) in group $\mathrm{I}$ and from $6.2 \pm 2.4$ to $2.7 \pm 2.6 \mathrm{~cm}$ (a decrease of $58 \pm 44 \%$, $\mathrm{p}=0.001)$ in group II. ANOVA showed a significant decrease in time for group II $(\mathrm{p}=0.005)$, but not for group I $(p=0.26)$. The percentage decrease after 10 days showed a slight, although not significant difference between the groups $(\mathrm{p}=0.086)$. When analysing 

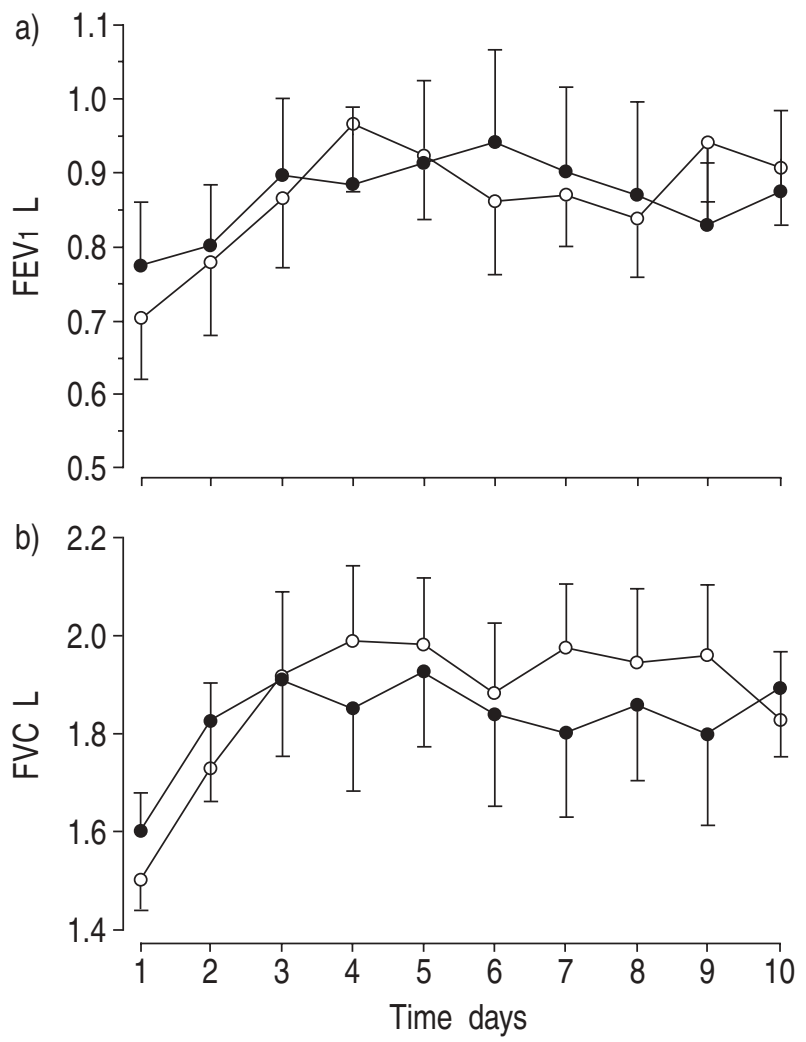

Fig. 1.-a) Forced expiratory volume in one second (FEV1) and b) forced vital capacity (FVC) versus time for group I ( $)$ and group II $(\bigcirc)$ during hospital stay. Data are presented as mean \pm SEM.

Table 2. - Effect of received treatment

\begin{tabular}{lccc}
\hline Value & Group I & Group II & p-value \\
\hline Subjects n & 23 & 25 & \\
Dropouts n & & & \\
$\quad$ Therapy failure & 3 & 3 & \\
$\quad$ Noncompliance & 0 & 3 & \\
$\quad$ Miscellanious & 1 & 1 & \\
FEV1 L & & & \\
$\quad$ On admission & $0.82 \pm 0.46$ & $0.70 \pm 0.27$ & 0.43 \\
At 10 days & $0.91 \pm 0.47$ & $0.90 \pm 0.29$ & 0.95 \\
At 4-week follow-up & $0.91 \pm 0.35$ & $1.31 \pm 0.40$ & 0.05 \\
Combined CRQ score & & & \\
$\quad$ On admission & $78 \pm 24$ & $67 \pm 17$ & 0.40 \\
At 4-week follow-up & $90 \pm 24$ & $86 \pm 20$ & 0.73 \\
Dyspnoea score VAS & & & \\
$\quad$ On admission & $6.0 \pm 1.9$ & $6.2 \pm 2.4$ & 0.76 \\
$\quad$ At 10 days & $4.1 \pm 2.6$ & $2.7 \pm 2.6$ & 0.15 \\
Patient satisfaction 1-5 & & & \\
$\quad$ On discharge & $4.69 \pm 0.6$ & $4.31 \pm 0.75$ & 0.14 \\
4-week follow-up & $4.75 \pm 0.87$ & $4.50 \pm 1.07$ & 0.57 \\
Length of hospital stay days & $15.5 \pm 10.3$ & $10.6 \pm 2.8$ & 0.06 \\
Cumulative steroid use mg & & & \\
14 day pre-admission & $98 \pm 89$ & $111 \pm 119$ & 0.74 \\
$\quad$ At 10 days & $356 \pm 67$ & $284 \pm 78$ & 0.005 \\
At discharge & $427 \pm 171$ & $309 \pm 101$ & 0.02 \\
Additional use & $36 \pm 67$ & $2 \pm 53$ & 0.10 \\
\hline
\end{tabular}

Data are presented as mean \pm SD unless otherwise stated. FEV1: forced expiratory volume in one second; CRQ: Chronic Respiratory Disease Index Questionnaire; VAS: visual analogue scale.

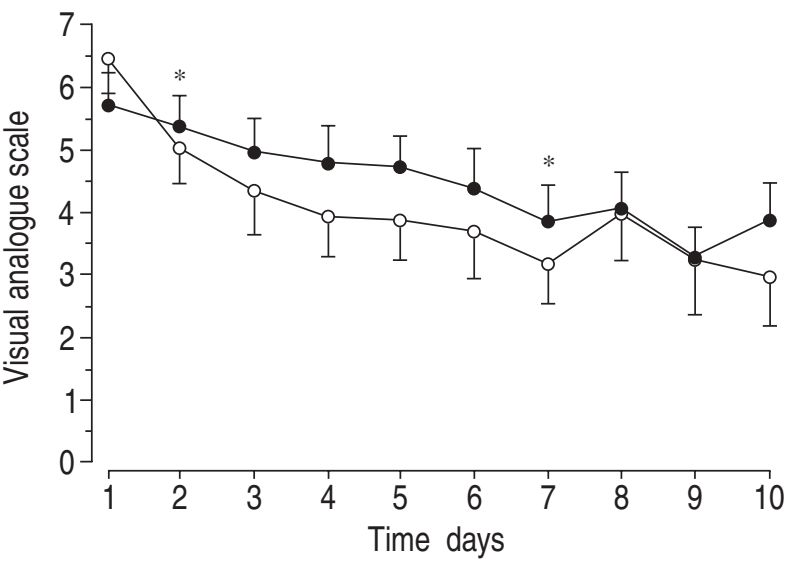

Fig. 2.-Dyspnoea versus time for group I ( $)$ and group II $(\bigcirc)$ during hospital stay. Data are presented as mean \pm SEM. *: $\mathrm{p}<0.05$.

the daily measured percentage decrease in dyspnoea scores, only day 5 revealed a significant difference between the two groups: $-14 \pm 42 \%$ for group I versus $-43 \pm 34 \%$ for group II $(\mathrm{p}=0.03)$. Comparing means at the 10 intervals revealed no statistical differences.

The means and mean percent changes in dyspnoea scores for group I and II during the 10 days of measurements are given in figure 2 and table 2.

\section{Quality of life and patient satisfaction}

The total CRQ scores to assess health related quality of life showed significant improvement between inclusion and 4-week follow-up for both groups: in group I, the CRQ rose from $78 \pm 24$ to $90 \pm 24$ points (increase of $20 \pm 33 \%, p=0.036$ ) and group II from $67 \pm 17$ to $86 \pm 20$ (increase of $45 \pm 50 \%$, $\mathrm{p}=0.0069)$. In group I a significant change was found in dyspnoea $(\mathrm{p}=0.038)$ and emotional functioning $(\mathrm{p}=0.053)$, whereas group II showed significant improvement in fatigue $(\mathrm{p}=0.022)$, emotional functioning $(\mathrm{p}=0.009)$ and mastery scores $(\mathrm{p}=0.020)$. Comparing the two groups, both the means and per cent changes in the four domains showed no statistical difference (ptot=0.19). The means and changes in CRQ scores are shown in figure 3 and table 2. As is shown on table 2, patient satisfaction with received treatment was comparably high in both groups and increased, though not significantly, after 4 weeks of follow-up.

\section{Re-admission rate}

Follow-up to assess the probability of re-admission lasted for 20 weeks. Figure 4 shows the percentage of patients not requiring hospital re-admission after discharge. Group II had a higher incidence of re-admissions, mainly in the first 6 weeks after discharge (eight re-admissions in group II versus four in group I). After 20 weeks $42 \%$ of the patients in group I had relapsed versus $66 \%$ of group II patients. Using the log-rank test to compare admission free survival 


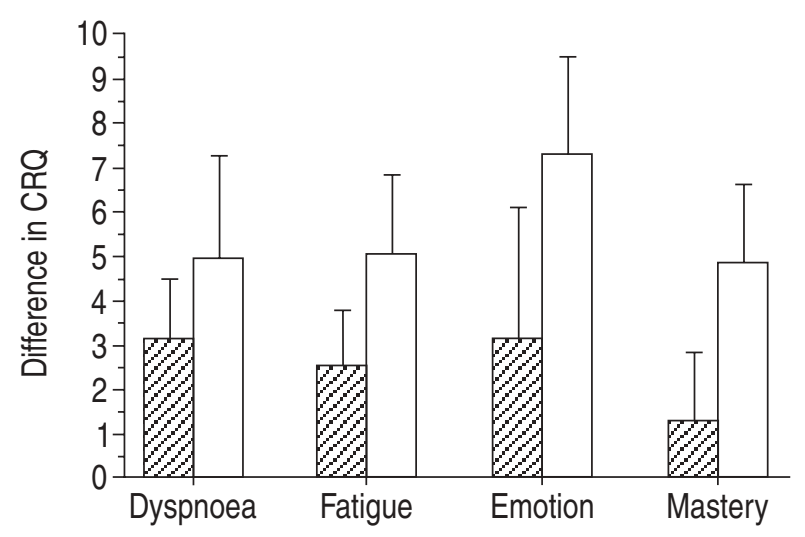

Fig. 3.-Difference of the Chronic Respiratory Disease Index Questionnaire (CRQ) score between 4-week follow-up and admission for dyspnoea, fatigue, emotional functioning and mastery for group $1(\mathbb{Z})$ and group $2(\square)$. Data are presented as mean \pm SEM.

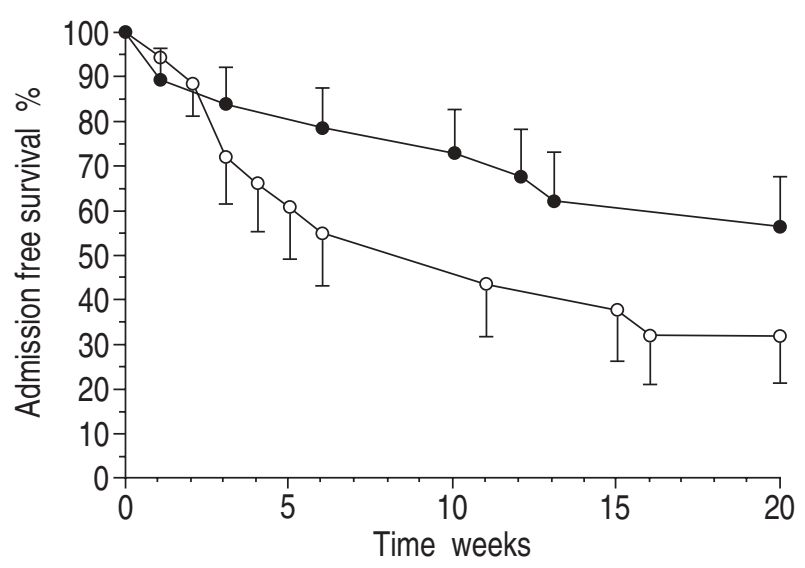

Fig. 4.-Admission free survival versus time for group I ( $)$ and group II $(\bigcirc)$ over a 20 -week period after discharge.

curves, no statistically significant difference was found $(\mathrm{p}=0.12)$.

\section{Use of medication}

Steroids. Ten-day cumulative systemic steroid intake (intravenous methylprednisolone for group I versus oral methylprednisolone for group II) differed significantly $(\mathrm{p}=0.005): 356 \pm 67 \mathrm{mg}$ in group I versus $284 \pm 78 \mathrm{mg}$ in group II. This could be attributed largely to the trial protocol, in which group I received a cumulative dose of $320 \mathrm{mg}$ of methylprednisolone and group II $296 \mathrm{mg}$, and partly due to the higher need for additional steroids in group I. As the physician in charge could increase or decrease steroids according to clinical improvement or deterioration, a supplemental steroid intake for group I of $36 \pm 66 \mathrm{mg}$ versus $2 \pm 53 \mathrm{mg}$ for group II $(\mathrm{p}=0.1)$ was noted.

The total cumulative systemic steroid intake at discharge differed significantly $(\mathrm{p}=0.02)$ and was higher in group I. This was partly due to the trial protocol and the higher need for additional steroids, and also due to an average longer hospital stay for group I: $16 \pm 10$ days versus $11 \pm 3$ days for group II $(\mathrm{p}=0.06)$.

\section{Bronchodilators}

Group I received aerosol therapy consisting of $10 \mathrm{mg}$ salbutamol.day ${ }^{-1}$ and $1 \mathrm{mg}$ ipratropiumbromide $\cdot$ day $^{-1}$, administered via four aerosols. This amounted to a possible 10-day cumulative dose of a $100 \mathrm{mg}$ salbutamol and $10 \mathrm{mg}$ ipratropiumbromide. Group II was given $1.6 \mathrm{mg}$ fenoterol and $640 \mu \mathrm{g}$ ipratropiumbromide in $4 \times 4$ puffs $\cdot$ day $^{-1}$, resulting in a planned cumulative dose of $16 \mathrm{mg}$ fenoterol and $6.4 \mathrm{mg}$ ipratropiumbromide. The attending physician, however, was free to change the dose of bronchodilators according to his judgement on the patient's progress. After 10-days, group I had received $94.6 \pm 9.8 \mathrm{mg}$ salbutamol $(-5 \pm 10 \%$, compared to protocol) and $9.5 \pm 0.98 \mathrm{mg}$ ipratropiumbromide $(-5 \pm 10 \%)$. Group II had received $13.9 \pm 3.2 \mathrm{mg}$ fenoterol $(-1 \pm 2 \%$, compared to protocol) and $5.6 \pm 1.3 \mathrm{mg}$ ipratropiumbromide $(-13 \pm 20 \%)$. Analysis showed no difference in extra intake of bronchodilators between the two groups $(\mathrm{p}=0.15)$.

\section{Discussion}

Numerous studies have confirmed that the use of systemic steroids and bronchodilators has an overall beneficial effect on the recovery of patients who are admitted for acute exacerbations of COPD [16]. In accordance, the present trial shows a similar improvement in spirometric measurements and symptom scores with this therapeutical treatment strategy. More importantly the present trial demonstrates that the observed effect is independant of the route of administration of steroids (intravenous or oral) and the device used for bronchodilation (aerosol or MDI). A 10-day inpatient regime of systemic steroids and bronchodilators resulted in an recovery of PFTs and a reduction in dyspnoea scores. After a period of 4 weeks the improvement in FEV1 and FVC was sustained and the CRQ scores had risen significantly. Since both strategies did not differ significantly in their effectiveness to treat COPD exacerbations, the authors conclude that the route or device by which the agents are administered does not result in a different clinical outcome. Although by protocol, group I received a higher dose of steroids and bronchodilators than group II, there was no difference in patient satisfaction with the received treatment or in the additional need for medication or length of hospital stay in the two groups. This suggests that the optimal dosage for steroids and bronchodilators is relatively arbitrary. However, whereas group I tended to have a longer hospital stay, group II showed a tendency towards a higher risk of re-admission 20 weeks after discharge.

Little research has been done to compare the combination of intravenous steroids and aerosol bronchodilators, to oral steroids and MDI bronchodilators. The present randomized trial is the first to 
investigate whether the two treatment regimes are equally effective in treating COPD exacerbations in an inpatient setting. The standard treatment used by the authors division (intravenous steroids and aerosol bronchodilators) and a frequently applied therapy in the outpatient management of exacerbations (oral steroids and MDI bronchodilators) were compared. The doses usually administered for each treatment regime in the authors division were applied, although these were slightly lower than in previous trials comparing systemic steroids to placebo [1-3].

A major limitation of the present study is the comparison of combination treatments. Because the two treatment regimes consisted of two components, steroids and bronchodilators, only conclusions on treatment as a whole can be drawn, disregarding which specific variable played a significant role in the observed changes. However, meta-analysis by TURNER et al. [9] and numerous other trials have shown that the different modes of administration of bronchodilators in the treatment of stable [10-12] and acute [9] COPD in in- and outpatient settings are associated with comparable therapeutic outcomes. To date, no trials have sufficiently evaluated the dose-response relationship to short-acting $\beta_{2}$-agonists in COPD exacerbations, or the comparison of salbutamol versus fenoterol as agents for bronchodilation. The present study does not show whether it is the administered dose or the agent which resulted in a different clinical outcome in the treatment of COPD exacerbations.

It could be argued that the relatively small number of patients might affect the results after the analysis of the obtained measurements. However power analysis performed to estimate sample size for the different outcome variables, demonstrated sufficient power for the difference in FEV1 and quality of life scores but not for the probability of re-admission with the number of included patients in the present trial. In retrospect, using a pooled standard deviation of $0.393 \mathrm{~L}$, power analysis revealed that each group would have had to consist of at least 24,400 patients to obtain a significant difference between the recorded 10-day FEV1 of group I $(0.91 \mathrm{~L})$ and II $(0.90 \mathrm{~L})$. It thus seems unlikely that a significant difference in FEV1 between the two treatment groups would be observed. Conclusions on the re-admission rate, however, have to be drawn with caution.

FEV1 measurements are a highly predictive factor in the clinical outcome of COPD exacerbations [20]. In contrast to other trials patients who had already received systemic steroids prior to hospital admission were not excluded. Nevertheless the degree of spirometric improvement registered in both of the treatment groups, $\pm 30 \%$ early improvement, is consistent with that found in other trials [9-10]. PFTs improved in both treatment groups, particularly in the first few days after onset of treatment. A similar evolution as seen in the trial conducted by NIEWOEHNER et al. [10] and SeEMUNGal et al. [21] was noted, since the improvement gradually declined and no significant improvement was seen when comparing the PFTs on discharge with those gathered at the 4-week follow-up visit.
The marginally larger increase in FEV1 in group II, could not be attributed to the higher dropout rate in that group. A higher dropout rate might exclude patients with more severe COPD and a potentially greater impairment of spirometric recovery. However, the number of dropouts merely due to therapy failure was equal in both groups, so this was unlikely to result in an exclusion of the sicker patients and a subsequent rise in mean FEV1 improvement in group II.

Because an increase in FEV1 does not necessarily correlate with subjective improvement, registering patient dyspnoea and CRQ scores may help in assessing the therapeutic effect of the administered treatment. In the present trial, both groups expressed a similar marked reduction in dyspnoea during the 10 -day treatment phase $(30 \%$ decrease in group I versus $60 \%$ in group II, $\mathrm{p}=0.09)$. This reduction continued during the follow-up period, as the CRQ scores in both groups showed a further parallel decline in the domain of dyspnoea after 4 weeks.

The overall improvement in the quality of life questionnaire, established 4 weeks after discharge, exceeded the minimal clinically important difference (10 points [17]) in both treatment groups. In group I the change was most noticeable in the dyspnoea domain $(\mathrm{p}=0.03)$, whereas for group II it was most evident for emotional functioning $(p=0.009)$. However, the change in quality of life was only assessed in patients who attended the 4-week follow-up visit. Therefore, not including the patients who died, had been readmitted or who refused to attend, could have lead to an overestimation in the rise in quality of life scores.

COPD patients, especially those with frequent exacerbations, are prone to multiple hospital admissions. This constitutes a significant problem in the COPD population. SEemungal et al. [22] suggested that quality of life in COPD patients correlates with the frequency of exacerbations and Osman et al. [23] showed that COPD patients with poor quality of life scores are more likely to be referred to respiratory specialists and have a greater risk of being re-admitted to hospital following an exacerbation. Although both groups expressed a significant increase in quality of life scores, re-admission rates were high in the patient population.

Numerous trials $[6,8]$ have established a decrease in the cumulative risk of immediate relapse after casualty treatment of exacerbations with systemic steroids as compared to placebo. The positive effect increased in patients who had been prone to multiple re-admissions in the past. In the patient population in this study, those on oral steroids showed a lower, though not significant, risk of relapse in the first 2 weeks after discharge compared to the intravenous group. After 2 weeks the relative risk in the oral group rose and surpassed that of the intravenous group during the continuation of the follow-up period. In the present study, as in previous studies [3, 19, 20], a very high re-admission rate after 20 weeks, $42 \%$ in group I and $67 \%$ in group II, was noted. The difference was not statistically significant $(p=0.12)$, although it might have been significant in a larger study population as the present study did not 
have sufficient power to draw conclusions on the re-admission rate (see earlier). Since both groups did not significantly differ in exacerbation severity or in the presence of co-morbidity on admission, the higher incidence of re-admission in the subset of patients on oral steroids is of concern. This higher incidence of re-admission could constitute an important limitation of an oral steroid regime. The present study, however, does not allow conclusions to be drawn on the factors associated with short- and long-term re-admission in COPD patients. Identification of treatment regimes that might be associated with higher re-admission rates is of great importance and thus it appears logical to direct future studies to this outcome.

In COPD patients, the majority of the costs are associated with exacerbations as these often necessitate hospital admissions [24, 25]. Therefore treatment that improves quality of life and limits hospital admission and relapse, constitutes an important element in reducing the impact of the disease on the patient's life and in tapering treatment costs. GRAVIL et al. [26] reported that many patients can be successfully treated at home after an initial clinical assessment in the hospital. The observations in this study enhance the potential for home treatment for a subset of patients referred to casualty for exacerbations of COPD. As pointed out by COENEN et al. [27] and Postma [28], further research should determine which population of COPD patients with an exacerbation can be treated at home equally successfully as in in-patient management, especially with regards to the risk of re-admission.

In conclusion, intravenous steroids and aerosol bronchodilators do not appear to be superior to oral steroids and metered dose inhaler bronchodilators when treating chronic obstructive pulmonary disease exacerbations in an inpatient setting. Both pharmacotherapeutical approaches are equally effective in the recovery of pulmonary function tests, improving subjective dyspnoea symptoms and health related quality of life. They are associated with an equal length of hospital admission and level of patient satisfaction. However, treatment with oral steroids and metered dose inhaler bronchodilators appears to be associated with a higher risk of hospital re-admission, warranting further clinical investigation.

\section{References}

1. Thompson WH, Nielson CP, Carvalho P, Charan NB, Crowley JJ. Controlled trial of oral prednisone in outpatients with acute COPD exacerbation. Am J Resp Crit Care Med 1996; 154: 407-412.

2. Davies L, Angus RM, Calverley PM. Oral corticosteroids in patients admitted to hospital with exacerbations of COPD: a prospective randomised controlled trial. Lancet 1999; 354: 456-460.

3. Niewoehner DE, Erbland ML, Deupree RH, et al. Effect of systemic glucocorticoids on exacerbations of COPD. N Eng J Med 1999; 340: 1941-1947.

4. Mitchell DM, Gildeh P, Rehahn M, Dimond AH, Collins JV. Effects of prednisolone in chronic airflow limitation. Lancet 1984; 2: 193-196.
5. Albert RK, Martin TR, Lewis SW. Controlled clinical trial of methylprednisolone in patients with chronic bronchitis and acute respiratory insufficiency. Ann Intern Med 1980; 92: 753-758.

6. Bullard MJ, Liaw SJ, Tsai YH, Min HP. Early corticosteroid use in acute exacerbations of chronic airflow obstruction. Am J Emerg Med 1996; 14: 139_ 143.

7. Renkema TE, Schouten JP, Koeter GH, Postma DS. Effects of long-term treatment with corticosteroids in COPD. Chest 1996; 109: 1156-1162.

8. Murata GH, Gorby MS, Chick TW, Halperin AK. Intravenous and oral corticosteroids for the prevention of relapse after treatment of decompensated COPD. Chest 1990; 98: 845-849.

9. Turner MO, Patel A, Ginsburg S, FitzGerald JM. Bronchodilator delivery in acute airflow obstruction. A meta-analysis. Arch Intern Med 1997; 157: 17361744.

10. Ikeda A, Nishimura K, Koyama H, et al. Comparison of the bronchodilator effect of salbutamol delivered via a metered-dose inhaler with spacer, a dry-powder inhaler and a jet nebulizer in patients with chronic obstructive pulmonary disease. Respiration 1999; 66: 119-123.

11. Mestitz H, Copland JM, Mcdonald CF. Comparison of outpatient nebulized vs metered dose inhaler terbutaline in chronic airflow obstruction. Chest 1989; 96: 1237-1240.

12. Jenkins SC, Heaton RW, Fulton TJ, Moxham J. Comparison of domiciliary nebulized salbutamol and salbutamol from a metered-dose inhaler in stable chronic airflow limitation. Chest 1987; 91: 804-807.

13. ATS Statement. Standards for the diagnosis and care of patients with COPD. Am J Respir Crit Care Med 1995; 152: S77-S120.

14. Guyat GH, Berman LB, Townsend M, Pugsley SO, Chambers LW. A measure of quality of life for clinical trials in chronic lung disease. Thorax 1987; 42: 773778.

15. American Thoracic Society. Standardization of Spirometry. Update. Am Rev Respir Dis 1987; 136: 12851298.

16. American Thoracic Society. Standardization of Spirometry. Update. Am J Respir Crit Care Med 1995; 152: $1107-1136$.

17. Redelmeier DA, Guyatt GH, Goldstein RS. Assessing the minimal important difference in symptoms: a comparison of two techniques. J Clin Epidemiol 1996; 49: $1215-1219$.

18. Troosters T, Gosselink R, Decramer M. Short- and long-term effects of outpatient rehabilitation in patients with chronic obstructive pulmonary disease: a randomized trial. Am J Med 2000; 109: 207-212.

19. Connors AF Jr, Dawson NV, Thomas C, et al. Outcomes following acute exacerbation of severe chronic obstructive lung disease. Am J Respir Crit Care Med 1996; 154: 959-967.

20. Niewoehner DE, Collins D, Erbland ML. Relation of FEV1 to clinical outcomes during exacerbations of chronic obstructive pulmonary disease. Department of Veterans Affairs Cooperative Study Group. Am J Respir Crit Care Med 2000; 161: 1201-1205.

21. Seemungal TA, Donaldson GC, Bhowmik A, Jeffries DJ, Wedzicha JA. Time course and recovery of exacerbations in patients with chronic obstructive 
pulmonary disease. Am J Respir Crit Care Med 2000; 161: 1608-1613.

22. Seemungal TA, Donaldson GC, Paul EA, Bestall JC, Jeffries DJ, Wedzicha JA. Effect of exacerbations on quality of life in patients with chronic obstructive pulmonary disease. Am J Respir Crit Care Med 1998; 157: 1418-1422.

23. Osman LM, Godden DJ, Friend JAR, Legge JS, Douglas JG. Quality of life and hospital re-admission in patients with chronic obstructive pulmonary disease. Thorax 1997; 52: 67-71.

24. Niederman MS, McCombs JS, Unger AN, Kumar A, Popovian R. Treatment costs of acute exacerbations of chronic bronchitis. Clin Ther 1999; 21: 576-591.
25. Price MJ, Hurrell C, Efthimiou J, Medley HV. Health care costs of treating exacerbations of chronic obstructive pulmonary disease (COPD). Eur Respir $J$ 1999; 14: Suppl. 30, 380s.

26. Gravil JH, Al-Rawas OA, Cotton MM, Flanigan U, Irwin A, Stevenson RD. Home treatment of exacerbations of chronic obstructive pulmonary disease by an acute respiratory assessment service. Lancet 1998; 351: 1853-1855.

27. Coenen S, Avonts D, Van Royen P. Chronic obstructive pulmonary disease: don't forget the gatekeeper. Lancet 1998; 352: 649.

28. Postma DS. When can an exacerbation of COPD be treated at home? Lancet 1998; 351: 1827-1828. 